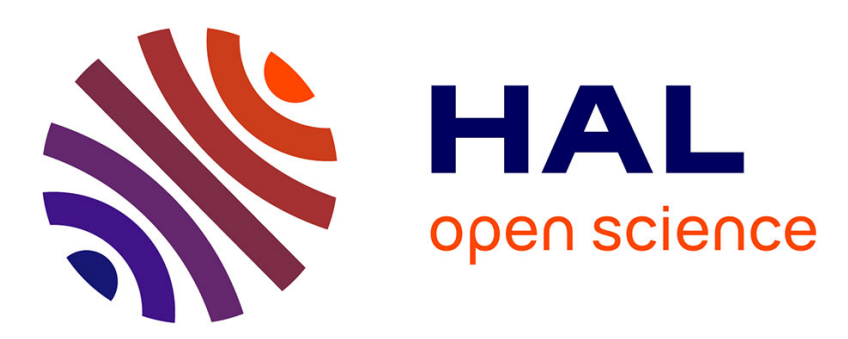

\title{
Shape coexistence in odd and odd-odd nuclei in the $\mathrm{A} \sim$ 100 region
}

\author{
J.A. Pinston, J. Genevey, G.S. Simpson, W. Urban
}

\section{To cite this version:}

J.A. Pinston, J. Genevey, G.S. Simpson, W. Urban. Shape coexistence in odd and odd-odd nuclei in the $\mathrm{A} \sim 100$ region. 3rd International Workshop on Nuclear Fission and Fission-Product Spectroscopy, Fission 2005, May 2005, Chateau de Cadarache, Saint Paul Lez Durance, France. pp.149-156, 10.1063/1.2137241 . in2p3-00024168

\section{HAL Id: in2p3-00024168 https://hal.in2p3.fr/in2p3-00024168}

Submitted on 26 May 2005

HAL is a multi-disciplinary open access archive for the deposit and dissemination of scientific research documents, whether they are published or not. The documents may come from teaching and research institutions in France or abroad, or from public or private research centers.
L'archive ouverte pluridisciplinaire HAL, est destinée au dépôt et à la diffusion de documents scientifiques de niveau recherche, publiés ou non, émanant des établissements d'enseignement et de recherche français ou étrangers, des laboratoires publics ou privés. 


\title{
Shape Coexistence In Odd And Odd-Odd Nuclei In The A 100 Region
}

\author{
J. A. Pinston ${ }^{1}$, J. Genevey ${ }^{1}$, G. Simpson ${ }^{2}$, and W. Urban ${ }^{3}$ \\ ${ }^{1}$ Laboratoire de Physique Subatomique et de Cosmologie, \\ F-38042 Grenoble Cedex, France \\ ${ }^{2}$ Institut Laue-Langevin, F-38042 Grenoble Cedex, France \\ ${ }^{3}$ Institute of Experimental Physics, Warsaw University \\ Ul. Hoza 69, PL-OO-681 Warzawa, Poland
}

\begin{abstract}
In the even-even nuclei around $A=100$ a transition from spherical to deformed shapes occurs from $N=58$ to $N=60$. The isotones with $N=59$ are of special interest, because they are just at the border between the two regions. Very recently, we have studied odd-neutrons and odd-odd nuclei with $N=59$, by means of prompt $\gamma$-ray spectroscopy of the spontaneous fission of ${ }^{248} \mathrm{Cm}$, using the EUROGAM 2 multi-detector, and by measurements of $\mu$ s isomers produced by fission of ${ }^{239,241} \mathrm{Pu}$ with thermal neutrons at ILL (Grenoble). In the latter case, the detection is based on time correlation measurements between fission fragments detected by the LOHENGRIN mass spectrometer and $\gamma$-rays or conversion electrons from the isomer decay. It was found that three shapes coexist in the odd ${ }^{97} \mathrm{Sr}$ and ${ }^{99} \mathrm{Zr}$ and two shapes coexist in the odd-odd ${ }^{96} \mathrm{Rb}$. A simple explanation of the shape-coexistence mechanism is proposed. It is based upon the Nilsson diagram and stresses the fundamental importance of the unique parity states.
\end{abstract}

Keywords: Exotic nuclei, Shape coexistence.

PACS: 23.20.Lv, 25.85.Ca, 25.85.Ec, 21.60.Ev

\section{INTRODUCTION}

The region of neutron-rich nuclei near $A=100$ is distinctive for the sudden change in the ground-state (g.s.) properties of nuclei. In particular, for the even ${ }^{98} \mathrm{Sr}$ and ${ }^{99} \mathrm{Zr}$ isotopes a sudden onset of strong deformation at $N=60$ is observed, whereas the lighter isotopes up to $N=58$ are rather spherical. Consequently, the isotones with $N=59$ are of special interest because they are just at the border between the two regions. A good knowledge of the spectroscopic properties of these nuclei should allow a better understanding of the origin of the deformation and the nature of the shape-coexistence phenomena in this mass region. Unfortunately, the $N=59$ isotones of interest are far from the stability line and are rather difficult to study. Consequently, for several decades, only $\beta$-decay experiments were performed and only low-spin states were measured. The main progress in this mass region was recently obtained by Urban et al. [1] who was able to observe for the first time well developed rotational bands in ${ }^{97} \mathrm{Sr}$ and ${ }^{99} \mathrm{Zr}$. In this work, the $N=59$ isotones were produced in the spontaneous fission of ${ }^{248} \mathrm{Cm}$ and the prompt $\gamma$-rays were measured using the EUROGAM $2 \mathrm{Ge}$ array. The level scheme of ${ }^{99} \mathrm{Zr}$ is shown in figure 1. These data confirmed that the g.s. and first 
two excited states are the neutron $\mathrm{s}_{1 / 2}, \mathrm{~d}_{3 / 2}$ and $\mathrm{g}_{7 / 2}$ shell model levels, but in addition, two well developed rotational bands, based upon the intrinsic configurations $v[411$ $\left.3 / 2^{+}\right]$and $v\left[5413 / 2^{-}\right]$, were also found at about $600 \mathrm{keV}$ excitation. The observation of these regular bands has firmly established the shape coexistence in these two $N=59$ isotones. More interesting, the quadrupole moments of these two bands were also measured and a mean deformation of $\beta_{2}=0.32(2)$ was deduced. It is interesting to note that this value is well below the deformation measured for the g.s. band in ${ }^{98} \mathrm{Sr}$ $\left(\beta_{2}=0.41(2)\right)[1]$, which is expected to be the maximum deformation in this region.

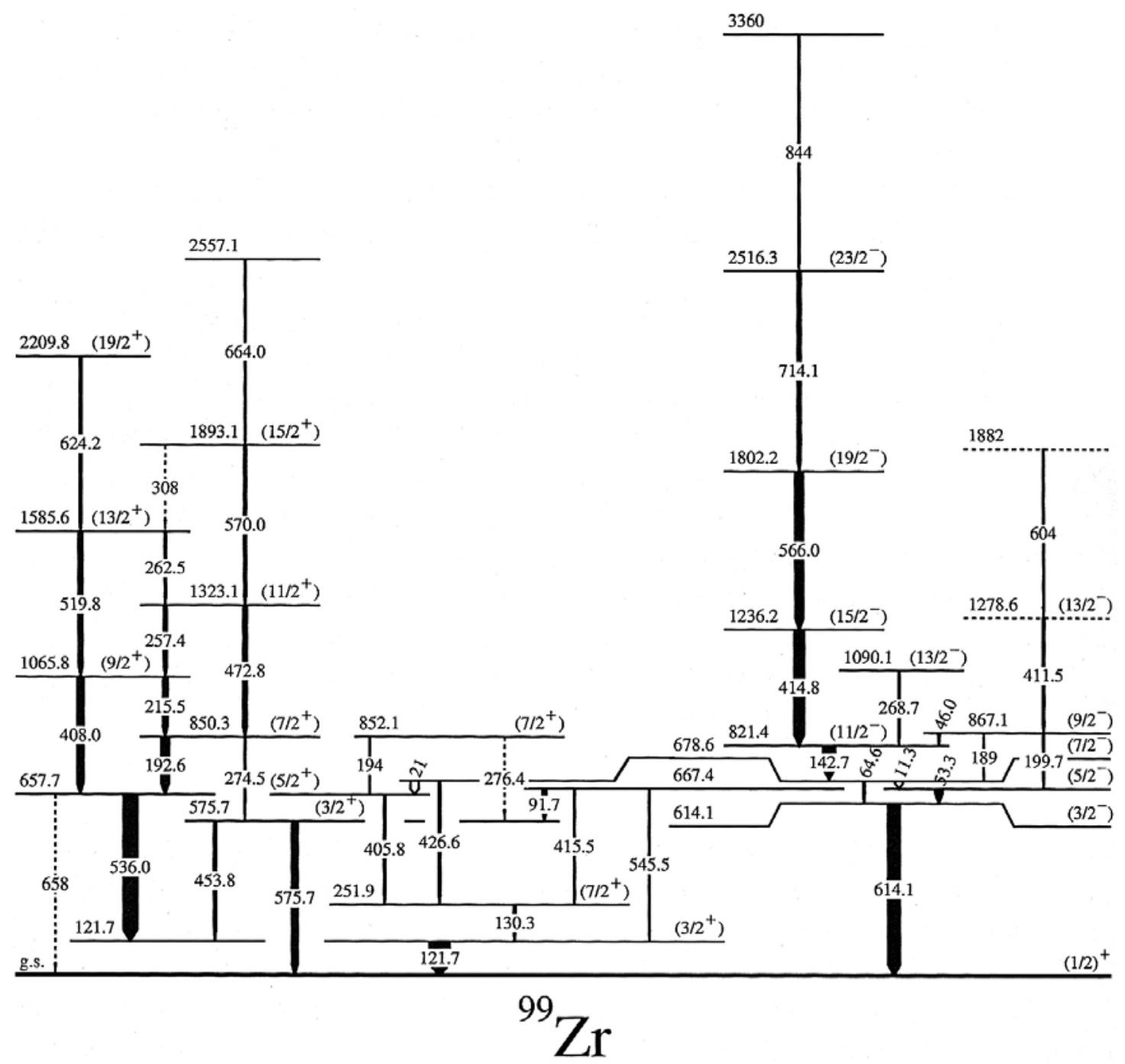

FIGURE 1. Level scheme of ${ }^{99} \mathrm{Zr}$ from EUROGAM 2 experiment. The $g . s$. and two first excited states are spherical, while two rotational bands based on $v\left[\begin{array}{lll}411 & 3 / 2^{+}\end{array}\right]$and $v\left[\begin{array}{lll}541 & 3 / 2^{-}\end{array}\right]$configurations, respectively, are present at about $500 \mathrm{keV}$ excitation (from [1]).

This first success triggered a more complete study of the nuclei of this mass region. For this purpose, a combination of two different techniques was used. In the first one, the nuclei of ${ }^{99} \mathrm{Zr}$, ${ }^{97} \mathrm{Sr}$ and ${ }^{101} \mathrm{Zr}$, were produced by spontaneous fission of ${ }^{248} \mathrm{Cm}$ and 
the prompt $\gamma$ rays were detected with the EUROGAM 2 array, while in the second one, microsecond isomers in ${ }^{95} \mathrm{Kr},{ }^{97} \mathrm{Sr}$ and ${ }^{96} \mathrm{Rb}$, were produced by fission of ${ }^{239,241} \mathrm{Pu}$ and studied with the LOHENGRIN spectrometer at ILL. In the last experiments, the detection is based on time correlation between fission fragments selected by the spectrometer and the $\gamma$ rays and conversion electrons from the isomers. More details on this experimental setup can be found in Ref. $[2,3]$.
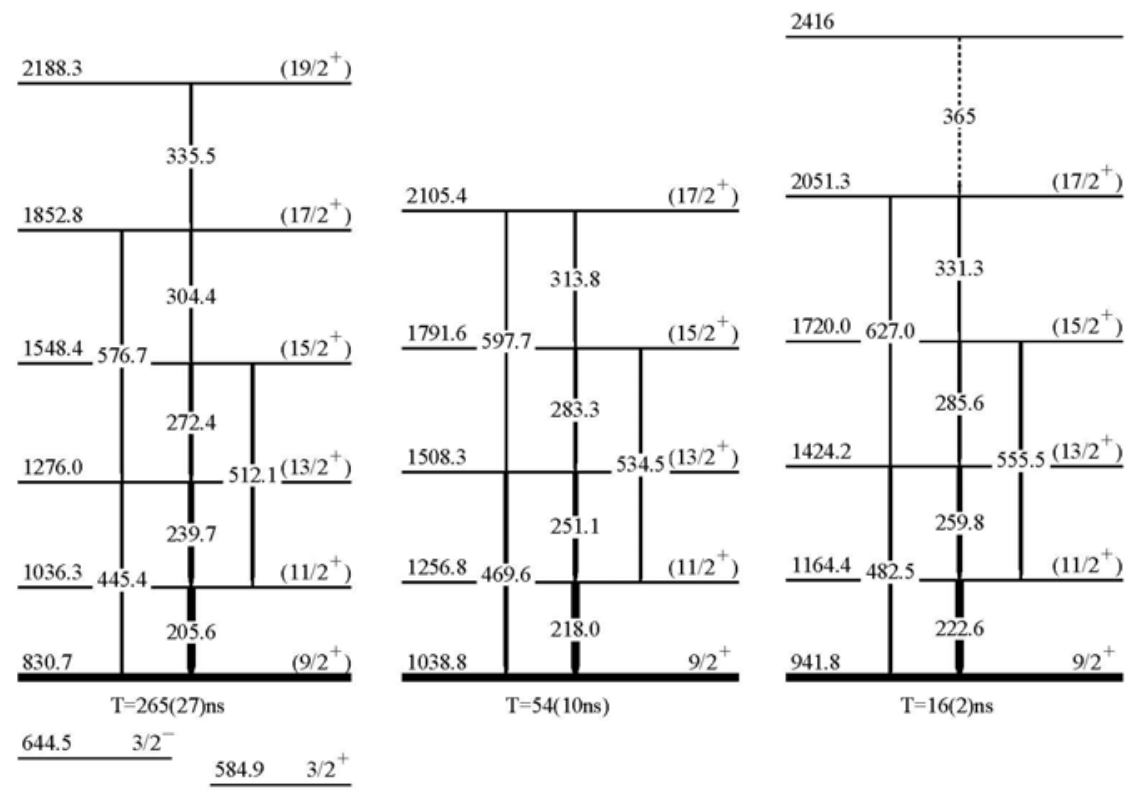

$644.5 \quad 3 / 2^{-}$
$584.9 \quad 3 / 2^{+}$

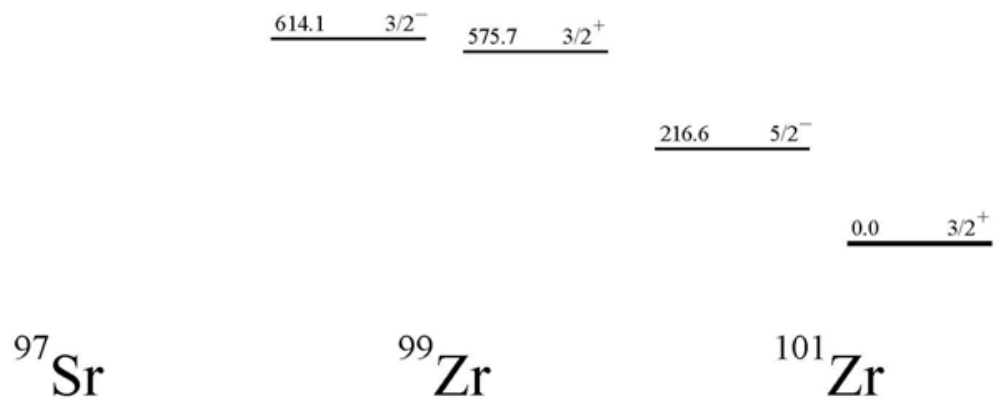

FIGURE 2. The level schemes of the strongly deformed $\left[4049 / 2^{+}\right]$bands in ${ }^{97} \mathrm{Sr},{ }^{99} \mathrm{Zr}$ and ${ }^{101} \mathrm{Zr}$. The levels below, mark the position of the other intrinsic configurations of moderate deformations (from $[5])$. 


\section{THE $\left[404\right.$ 9/2 ${ }^{+}$BAND IN ${ }^{99} \mathrm{ZR},{ }^{97}$ SR AND ${ }^{101} \mathrm{ZR}$}

A third rotational band was latter observed in ${ }^{99} \mathrm{Zr}$ from the data of EUROGAM 2 experiments [4]. The band head at $1038.8 \mathrm{keV}$ excitation is a $K$ isomer with a half life $T_{1 / 2}=54 \mathrm{~ns}$, which decays to several lower energy states. The spin and parity assignment of the band g.s., $I^{\pi}=9 / 2^{+}$, was derived from the intensities of the various partial branching ratios and angular-correlation measurements. For the first time, the [404 $\left.9 / 2^{+}\right]$band was observed in this mass region. Soon after, this band was also observed in ${ }^{97} \mathrm{Sr}[5,6,7]$ and ${ }^{101} \mathrm{Zr}[5]$. In these three nuclei, the band head is a $K$ isomer. However, for ${ }^{97} \mathrm{Sr}$, where the half life was re-measured very recently, the new value found (526(13) ns) was substantially longer than the one reported by Hwang et al. (265(27) ns) [6].

In figure 2 , the level schemes of the three $\left[4049 / 2^{+}\right]$bands are shown and the intrinsic excitations of the $3 / 2^{+}, 3 / 2^{-}$and $5 / 2^{-}$levels, of moderate deformation, are also indicated [5]. The intraband-transition energies show very strong similarities in these three nuclei and the energy spacings in these bands have a strongly coupled character. The quadrupole moments $Q_{0}$ deduced from the experimental $\gamma$ branching ratio $\Delta I=1$ to $\Delta I=2$ for intraband transitions lead to a mean deformation of $\beta_{2}=0.41(3)$, for these three nuclei.

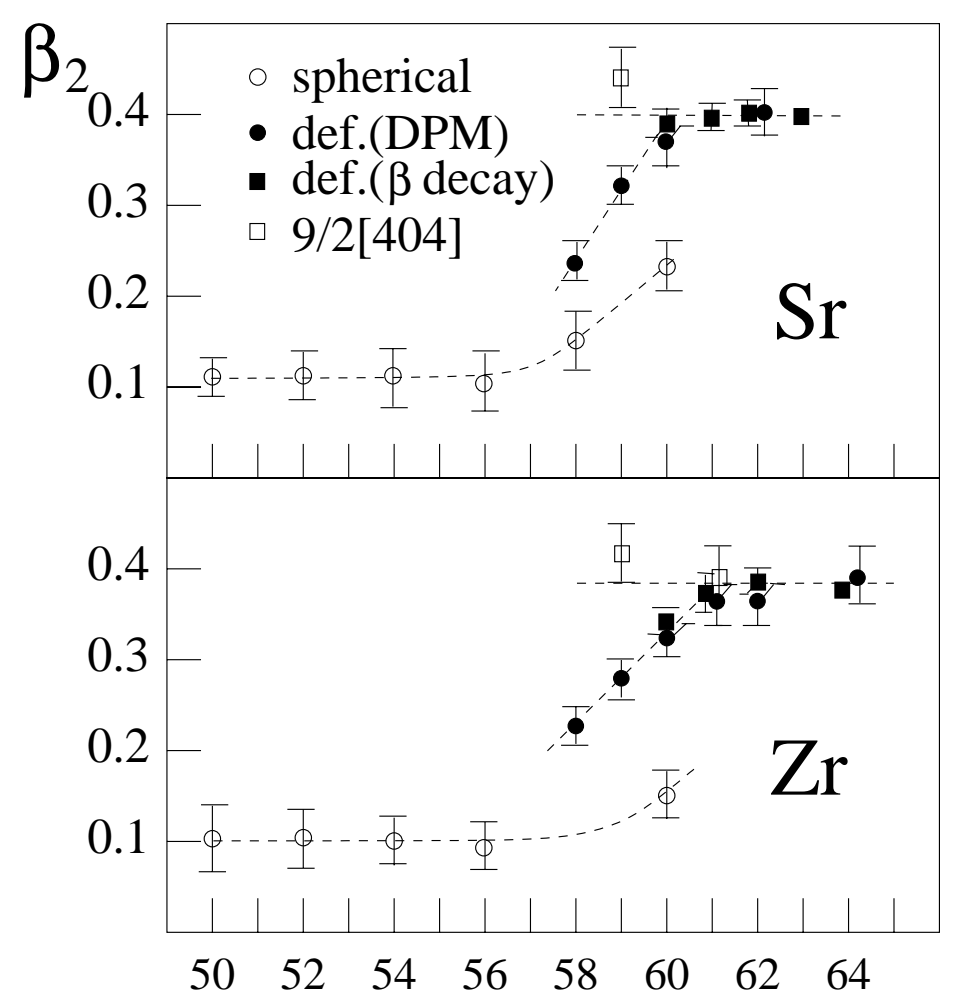

FIGURE 3. Systematic of deformations for various configurations in $\mathrm{Sr}$ and $\mathrm{Zr}$ isotopes. For $N=59$, isotones three shape coexist simultaneously. Above $N=59$, the deformation saturates at a value $\beta_{2}=0.41$. Note that this value is comparable to the deformation found for the $\left[4049 / 2^{+}\right]$band in ${ }^{97} \mathrm{Sr},{ }^{99} \mathrm{Zr}$ and ${ }^{101} \mathrm{Zr}$ (from [5]). 


\section{SHAPE COEXISTENCE IN $N=59$ ISOTONES}

In figure 3, the $\beta_{2}$ values for the three known $\left[4049 / 2^{+}\right]$bands in ${ }^{99} \mathrm{Zr}$, ${ }^{97} \mathrm{Sr}$ and ${ }^{101} \mathrm{Zr}$ are compared with the values found for the other bands in strontium and zirconium isotopes in $A=100$ mass region [5]. This picture shows that three shapes coexist for $N=59$ isotones. In these nuclei, the ground state and first two excited states are the neutrons $\mathrm{s}_{1 / 2}, \mathrm{~d}_{3 / 2}$ and $\mathrm{g}_{7 / 2}$ shell-model levels. At about $600 \mathrm{keV}$, two rotational bands, based upon the $v\left[4113 / 2^{+}\right]$and $v\left[5413 / 2^{-}\right]$configurations are present and have a mean deformation $\beta_{2}=0.32(2)$. In contrast, the $\left[4049 / 2^{+}\right]$bands at about $1 \mathrm{MeV}$ are strongly deformed and their deformations are comparable with the maximum value found in this mass region $\beta_{2}=0.41(2)$. This large value is observed for several even and odd $\mathrm{Sr}$ and $\mathrm{Zr}$ nuclei above $N=59$ and is remarkably constant.

The two-neutron unique-parity states, $\nu \mathrm{g}_{9 / 2}$ of the $N=4$ shell and $v \mathrm{~h}_{11 / 2}$ of the $N=5$ shell, play a dominant role in the mechanism of shape coexistence observed in this region. Figure 4 shows a portion of the Nilsson diagram, around $N=58$ region [5]. It is interesting to note that the occupation of the $\left[4049 / 2^{+}\right]$orbital, with its strong upwards slope, will favor a spherical equilibrium shape, whereas the state originating from the $\mathrm{g}_{9 / 2}$ level slopes downward and thus favors deformation. For the moderate deformation

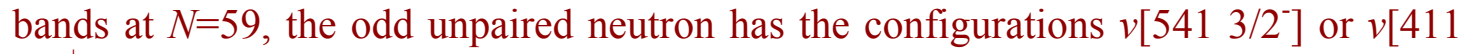
$\left.3 / 2^{+}\right]$. Consequently, in both bands the core has the same structure with one pair in the deformation-driving $v\left[\begin{array}{cc}550 & 1 / 2^{-}\end{array}\right]$orbital and the other pair in the up-sloping $v\left[\begin{array}{lll}404 & \left.9 / 2^{+}\right]\end{array}\right.$ orbital, working against deformation. This configuration produces the moderate deformation measured in ${ }^{99} \mathrm{Zr}$ and ${ }^{97} \mathrm{Sr}$ nuclei.

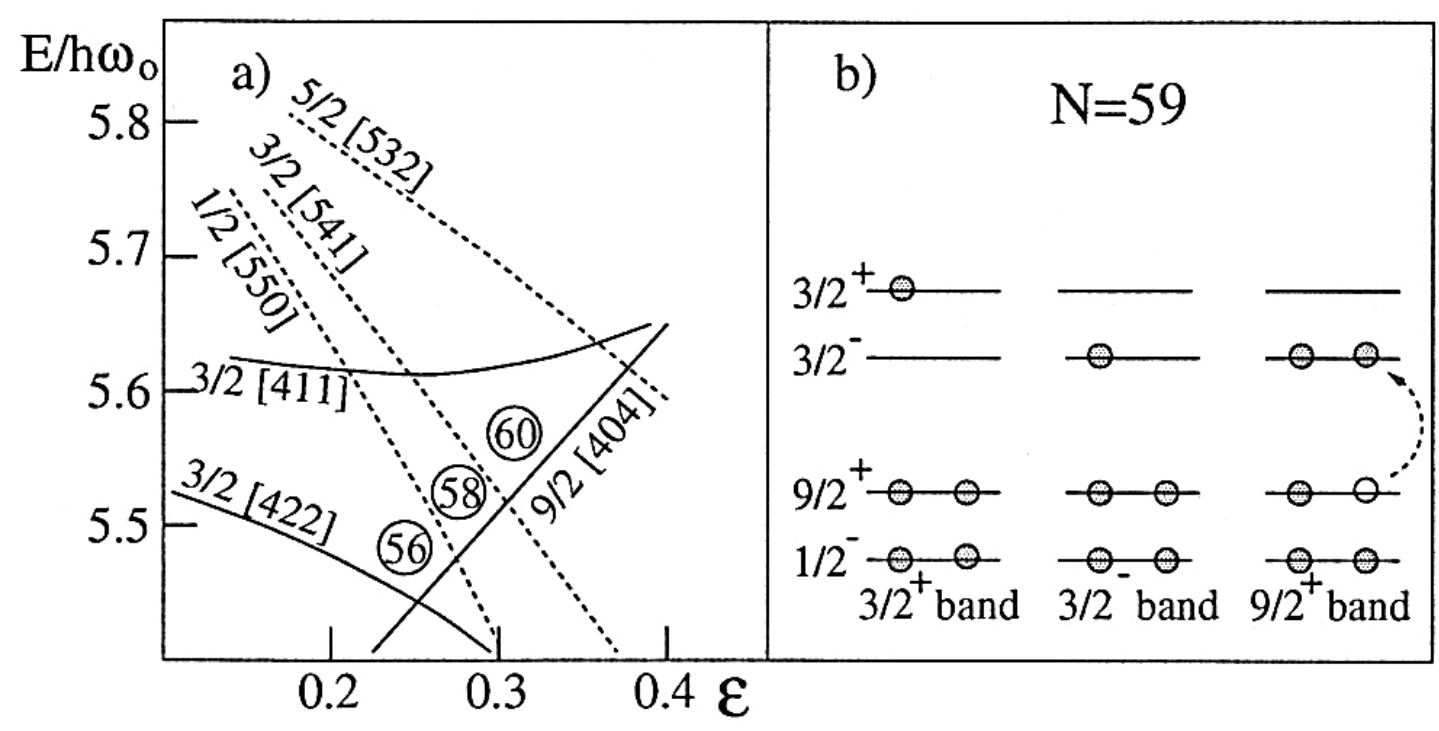

FIGURE 4. a) Portion of the Nilsson neutrons levels, b) Schematic representation of the deformed configurations in $\mathrm{Sr}$ and $\mathrm{Zr}$ isotopes (from[5]).

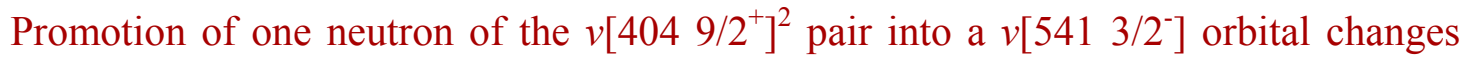
dramatically the situation. In this case, the core has four neutrons occupying the down 


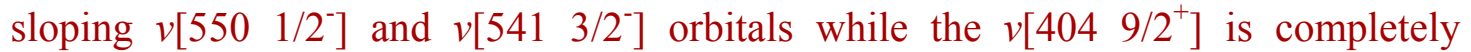
depleted. Now, the neutron spectator orbiting around the core is in the $v\left[4049 / 2^{+}\right]$ orbital. In this mass region, this configuration produces the deformation limit, already at $N=59$. A comparable mechanism was proposed a long time ago by Kleinheinz et al., $[8,9]$ to explain shape coexistence in the lanthanide region. In this case, the unique parity neutron states $v \mathrm{~h}_{11 / 2}$ and $v \mathrm{i}_{13 / 2}$ play a role analogous to the one of $v \mathrm{~g} 9_{/ 2}$ and $v \mathrm{~h}_{11 / 2}$ in the $A=100$ region. However, it is interesting to note that the effect is simpler in the latter region because just a fewer levels are active and the deformation change is also more dramatic.

\section{SHAPE COEXISTENCE IN ODD-ODD NUCLEI}

To increase nuclear-structure information in the $N=59$ isotones, the odd-odd nuclei were also investigated. For this reason, ${ }^{98} \mathrm{Y}$ was recently revisited by Brant et al. [10] and we have also reinvestigated ${ }^{96} \mathrm{Rb}$. The latter nucleus was previously measured with the LOHENGRIN spectrometer [11]. However, the efficiency of the $\gamma$ detection was too weak to build a reliable level scheme. More recently, this efficiency was strongly improved and the $\gamma$ and conversion electrons de-exciting the isomer were studied. The new level scheme is shown in figure 5. This very neutron-rich nucleus has a structure comparable to the previously known ${ }^{98} \mathrm{Y}$. Both nuclei show rather spherical levels at low energy, while deformed states appear at about $500 \mathrm{keV}$ excitation. The $\left(\pi\left[\begin{array}{ll}431 & 3 / 2^{+}\end{array}\right]\left[\begin{array}{lll}541 & 3 / 2^{-}\end{array}\right] 3^{-}\right.$and $\left(\pi\left[\begin{array}{lll}422 & 5 / 2^{+}\end{array}\right] v\left[\begin{array}{lll}541 & 3 / 2^{-}\end{array}\right]\right) 4^{-}$intrinsic configurations were found for ${ }^{96} \mathrm{Rb}$ and ${ }^{98} \mathrm{Y}$ nuclei, respectively. All these neutron and proton orbitals originate from the $\pi \mathrm{g}_{9 / 2}$ and $v \mathrm{~h}_{11 / 2}$ spherical unique parity states. A lower limit, $\beta_{2}>0.28$, was deduced for these two nuclei, from the experimental $\gamma$ branching ratio $\Delta I=1$ to $\Delta I=2$ for intraband transitions. Moreover, the comparable behavior observed for the decay of the isomer in both nuclei, suggests that they have the same $\left(\pi \mathrm{g}_{9 / 2} v \mathrm{~h}_{11 / 2}\right) 10^{-}$configuration. Consequently, in the same nucleus, these unique-parity states are present in spherical and deformed configurations. The presence of a spherical yrast trap in competition with spherical levels is the consequence of a strongly attractive $n-p$ interaction, because $n$ and $p$ are in coplanar orbitals. Very recently, we observed an $I^{\pi}=17^{-}$isomer in ${ }^{98} \mathrm{Zr}$ [12], of spherical origin and about 6.5 MeV excitation. This result shows that the competition between spherical and deformed states, along the yrast line, is still active at high excitation energy. 


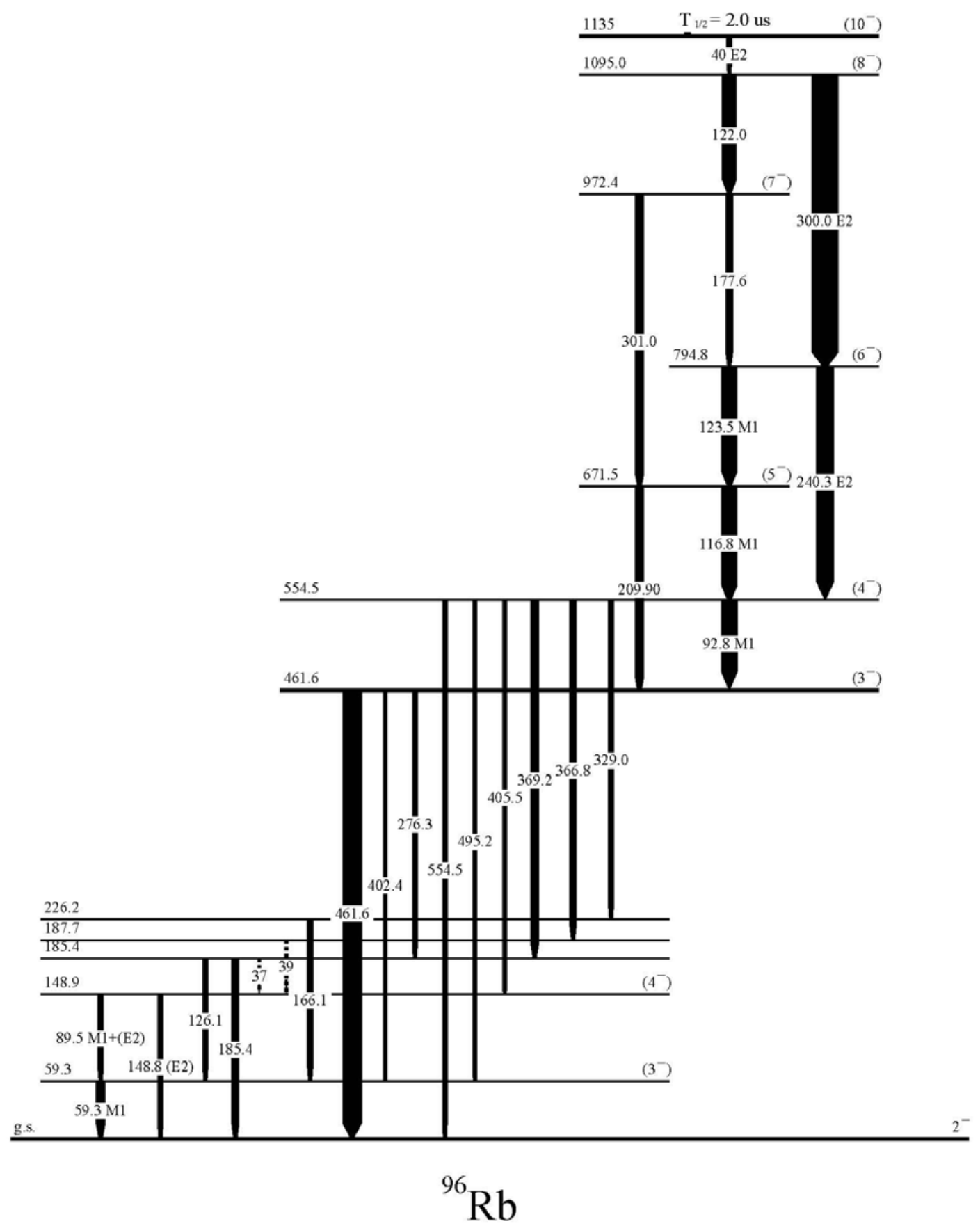

FIGURE 5. Level scheme of the $2.0 \mu$ s isomer in ${ }^{96} \mathrm{Rb}$. The g.s. with spin and parity $I^{\pi}=2^{-}$, the lowlying levels and the $1135 \mathrm{keV}$ isomer have rather spherical configurations, while a well developed rotational band is seen at $461.6 \mathrm{keV}$ excitation. 


\section{CONCLUSIONS}

In this work odd and odd-odd $N=59$ istones were investigated, using a combination of two experimental techniques. It is now well established that three shapes coexist in odd ${ }^{97} \mathrm{Sr}$ and ${ }^{99} \mathrm{Zr}$, while two different shapes were seen in odd-odd ${ }^{98} \mathrm{Y}$ and ${ }^{96} \mathrm{Rb}$. These new data demonstrate that the spectroscopy of odd- $A$ and odd-odd nuclei, provides much more information on the structure of the different shapes than eveneven nuclei. The theoretical interpretation of these collective excitations is based on the Nilsson diagram in the $N=58$ region and it shows that the spherical unique-parity state plays a very important role in the shape coexistence mechanism. In conclusion, a great wealth of nuclear structure information was recently gained for these odd and odd-odd $N=59$ isotones and we hope that these new results will trigger new calculations for this mass region.

\section{ACKNOWLEDGMENTS}

We thank Pr. Asghar for stimulating discussions and a careful reading of the manuscript.

\section{REFERENCES}

1. Urban W. et al., Nucl. Phys. A 689, 605 (2001).

2. Pinston J. A. and Genevey J., J. Phys. G 30, R57 (2004).

3. Genevey J. et al., Eur. Phys. J. A 9, 191 (2000).

4 Urban W. et al., Eur. Phys. J. A 16, 11 (2003).

5. Urban W. et al., Eur. Phys. J. A 22, 241 (2004).

6. Hwang J.K. et al., Phys. Rev. C 67, 054304 (2003).

7. Wu C. Y. et al., Phys. Rev. C 70, nn064312 (2004).

8. Kleinheinz P. et al., Phys. Rev. Lett. 32, 68 (1974).

9. Kleinheinz P. et al., Nucl. Phys. A 283, 189 (1977).

10. S. Brant et al., Phys. Rev. C 69, 034327 (2004).

11. Genevey J. et al., Phys. Rev. C 59, 82 (1999).

12. Simpson G. et al., talk presented at this conference. 